\title{
Banca pública, seguridad social y acceso a financiamiento de vivienda en Ecuador
}

\author{
Public banking, social security \\ and access to house financing in Ecuador
}

\author{
Katiuska King Mantilla \\ Universidad Central del Ecuador \\ kkking@uce.edu.ec \\ https://orcid.org/0000-0002-4704-3919
}

\begin{abstract}
Resumen
En el Ecuador, la seguridad social no es universal. El Banco de la Seguridad Social (Biess) se creó en 2009 y entró a operar formalmente un año después. Este Banco utiliza recursos de las reservas de la seguridad social para otorgar, entre otros, créditos hipotecarios para la vivienda de los afiliados. De esta forma, las reservas obtenían un mejor rendimiento que los obtenidos en títulos valores de hasta tres puntos y se otorgaban más beneficios a los cotizantes.

Esta política impulsó la construcción inmobiliaria en el país, incrementó la liquidez en la economía real y redujo las tasas de interés en el crédito de vivienda en 2,22 puntos porcentuales debido a que la provisión directa por parte del Biess generó mayor competencia y además los cotizantes podían pasar su crédito privado al banco de los afiliados. La vivienda propia entre los afiliados de la seguridad social aumentó en casi cuatro puntos entre el 2007 y el 2018. Los hogares con personas afiliadas que más cambiaron su participación en la tenencia de vivienda propia fueron los quintiles 2 y 3 .

La presencia del Biess y la compra de cartera modificó la operación crediticia en todo el segmento de vivienda. Este artículo realiza un balance de los resultados de la provisión directa a través de un operador público como forma alternativa de regulación financiera y a la tasa de interés en particular.
\end{abstract}

\section{Palabras clave}

Financiamiento de la vivienda, seguridad social, regulación financiera alternativa, finanzas, política financiera, política económica, provisión directa.

Forma sugerida de citar: King Mantilla, K. (2019). Banca pública, seguridad social y acceso a financiamiento de vivienda en Ecuador. Universitas, 31, pp. 119-139. 


\begin{abstract}
Social Security in Ecuador is not universal. The Ecuadorian Social Security Bank (Biess) was legally established in 2009, and began to operate formally one year later. The Biess uses social security resources to offer housing mortgages to its members: obtaining financial returns up to three percentage points higher, while at the same time offering more benefits to its affiliates.

The policy boosted housing construction, increased liquidity in the real economy and reduced interest rates on housing loans by 2.2 percentage points, as direct provision by Biess produced more competition and social security affiliates were able to transfer their privately financed mortgages to the Biess. House ownership among social security affiliates increased by almost four points between 2007 and 2018 . The households who most changed their participation from owning their own homes were located in quintiles 2 and 3. The presence of the Biess and its portfolio purchases modified financial regulation in the housing sector. This article takes stock of the results of direct provision by means of a public operator as an alternative form of financial regulation and of interest rates in particular.
\end{abstract}

\title{
Keywords
}

Housing finance, social security, alternative financial regulation, finance, financial policy, economic policy, direct provision.

\section{Introducción}

Los sistemas de seguridad social representan enormes recursos cuya inversión bien canalizada puede servir para fines redistributivos, económicos y sociales. Luego de la crisis mundial del 2008 quedó en evidencia la fragilidad de los sistemas financieros y la necesidad de su regulación.

Si bien la presencia de operadores públicos en los mercados financieros había sido materia de controversias, no se deja de reconocer que mejora la eficiencia de la banca privada y puede reducir la prociclidad del crédito (Micco, Panizza, \& Levy, 2007).

Ecuador en los primeros años de la dolarización, mantenía una política económica de no intervención en los mercados. En ese marco, el país en el año 2007 retoma los procesos de planificación venidos a menos desde los años noventa y construye un primer plan de desarrollo para el período 20072010 (Senplades, 2007) y luego de aprobada la Constitución, mediante re- 
feréndum, plantea otro plan para el período 2009-2013. En los dos planes consta el objetivo 11 "Establecer un sistema económico social, solidario y sostenible” (Senplades, 2007, p. 254; 2009, p. 329) y en el segundo Plan, aparece la política 11.13 "Promover el ahorro y la inversión nacionales, consolidando el sistema financiero como servicio de orden público, con un adecuado funcionamiento y complementariedad entre sus sectores público, privado y popular solidario" (2009, p. 339) al que el primer plan estratégico institucional del Biess se articulaba directamente (Biess, 2011, p. 3) y de forma más referencial en el segundo (Biess, 2017, p. 3).

En la nueva Constitución (Asamblea, 2008, pp. 38-39) la seguridad social aparece como un derecho y deber primordial del Estado, el artículo 34 define que se guía por los principios de solidaridad y universalidad.

Esping-Andersen (1990 citado en Cichon et al., 2006, p. 167), clasifica a los regímenes de estados de bienestar en tres, liberal, conservador o corporativista y social democrático. Tanto el liberal como el social democrático ofrecen coberturas universales mientras que el conservador lo hace solo a las personas empleadas. El Ecuador se situaría en el segundo tipo de régimen de bienestar y la Constitución plantea ir hacia el tercero.

Este artículo es parte de una investigación más amplia e inicia con un estudio de algunos resultados del Biess en base a la información disponible que será detallada más adelante. El uso de sus reservas es clave por su rol en la economía.

La Constitución también estableció en el artículo 372 (2008, p. 211) que las inversiones de la seguridad social se conducirán a través de una institución financiera cuyo propietario será el IESS para "generar empleo y valor agregado" (2008, p. 253).

En consecuencia en mayo del 2009, unos meses después de las crisis económica mundial, en Ecuador se aprueba la Ley de creación del Banco de la Seguridad Social (Biess) o Banco de los afilados (DNTI, 2009) como una institución financiera pública que puede realizar operaciones de banca de inversión y oferta servicios financieros específicos entre los que se cuenta créditos hipotecarios a sus afiliados y "operaciones de redescuento de cartera hipotecaria de instituciones financieras [IFI's]" (2009, p. 2).

La creación del Biess formaba parte de la nueva arquitectura financiera (Senplades, 2009, p. 78) sin un rol explícito de regulación. Sin embargo, luego de casi diez años de la puesta en marcha del Biess no existen investigacio- 
nes respecto al rol que tuvo en el mercado financiero porque no se tenía previsto que funcionara como un dispositivo de regulación financiera alternativa.

En este marco el presente artículo busca cubrir esta falencia y se plantea como hipótesis que la presencia del Biess en el sistema financiero introdujo una forma de regulación alternativa que redujo la tasa de interés del segmento hipotecario y aumentó el porcentaje de hogares con vivienda propia en el país.

El artículo está organizado en cinco secciones, la primera, en la que nos encontramos, que introduce el tema y el marco legal de creación de la nueva institucionalidad. La segunda, que realiza un estado del arte sobre el Biess, describe el marco teórico y la metodología utilizados. Los resultados se presentan en la tercera sección. En la cuarta se discute esta forma de regulación alternativa en el sistema financiero. En la quinta y última sección, se exponen las conclusiones.

\section{Marco teórico y metodología}

Tal como fuera introducido en la sección anterior, la irrupción de una nueva institución financiera pública en el Ecuador por mandato constitucional generó un cambio en el espectro financiero nacional a través de la composición del mercado hipotecario, la tasa de interés efectiva y el acceso a la vivienda propia.

Existen algunas investigaciones sobre el Biess, una de Carrillo (2013) sobre la influencia de los créditos hipotecarios con recursos de la seguridad social en la construcción en el período 2008-2012, encontrando causalidad al $90 \%$ de confianza entre los préstamos y la proyección de edificaciones en $\mathrm{m}^{2}$ (2013, p. 110). Ordóñez (2015) utiliza el mismo período temporal para realizar un análisis de series de tiempo de los permisos de construcción y concluye que sin la presencia del Biess, la construcción hubiese tenido un mayor dinamismo sin determinar causalidad y sin negar la "contribución del BIESS al crecimiento del sector inmobiliario" (2015, p. 95). Serrano Cueva y Naranjo Peñaherrera (2014) realizan una crónica de la creación del Biess y de su gestión del 2009 al 2014.

Deere y Catanzarite (2017) analizan la relación entre fuentes de crédito y la finalidad del mismo como medio para perpetuar la desigualdad en el Ecuador. Este estudio se realiza para el 2010 en base a una encuesta específica y se concluye que el sistema crediticio reproduce condiciones de des- 
igualdad y consideran al Biess como el banco público más grande en créditos a particulares (2017, p. 124).

Por otro lado, la CAF en un informe elaborado por Sanguinetti et al. (2011) a raíz del rol que tuvo la banca pública en la crisis económica mundial, discute los elementos que justifican su intervención y algunos factores que podrían definir su éxito. Pone énfasis en las instituciones denominadas banca de desarrollo que es la categoría a la que el Biess pertenece, así como, en el gobierno corporativo como factor de éxito.

Luego de casi diez años de la puesta en marcha del Biess, es posible realizar un estado de situación de algunos resultados en el segmento hipotecario donde la institución actuó de dos formas, como operadora directa y a través de operaciones de redescuento en la compra de cartera a IFI's privadas. Sin embargo, más que hablar de la institución del Biess, el objetivo del artículo es indagar formas alternativas de regulación en base a esta experiencia ecuatoriana con un operador directo con recursos de las reservas de la seguridad social.

Este artículo representa en palabras de Stiglitz (2000, p. 107) una investigación positiva de las consecuencias de dicha política. Los mecanismos de intervención pública de acuerdo a Subirats, Knoepfel, Larrue, y Varonne (2008, pp. 158-159) se agrupan en cuatro vías principales: reguladora, incentivadora, persuasiva, así como la provisión directa. Por la vía reguladora se busca generar cambios de comportamientos en los grupos objetivo, este camino incluye obligaciones o prohibiciones objeto de sanciones en caso de incumplimiento. La senda de los incentivos plantea una intervención más indirecta que opera mediante transferencias para incidir en el comportamiento de los grupos objetivo a través de impuestos o subvenciones (2008, p. 158). La vía persuasiva buscar convencer a los grupos objetivo para adoptar conductas en los sentidos que la política persigue. Mientras que la provisión directa muestra e induce la conducta esperada de los grupos objetivo (2008, p. 159).

En la vía reguladora en el ámbito financiero existen diversas formas de regular un mercado, a través de actos administrativos que definen lineamientos generales o parámetros específicos para la calidad de los servicios ofertados, la adopción de un conjunto de regulaciones internacionales como los Acuerdos de Basilea que marcan algunas medidas de apalancamiento, liquidez y capital, entre otros o estableciendo precios máximos que garanticen una tasa de rendimiento "justa". En caso de que no se cumplan estas nor- 
mas se requiere de control por parte de los entes supervisores. Ello implica contar con recursos humanos que posean las competencias y puedan hacer frente a habilidades de los regulados. Pese a ser la regulación más común, en la práctica resulta poco oportuna y eficaz en detectar o prevenir comportamientos dolosos, dado que desde que se crean las normas, las instituciones objeto de control ya empiezan a estudiar cómo las regulaciones pueden ser evitadas.

En términos económicos, una forma alternativa y menos común de intervención es a través de la provisión directa con uno o varios operadores públicos que a su vez influyan en el mercado y estimulen la competencia. Evidentemente el o los operadores públicos deben cambiar el comportamiento prevaleciente en el mercado, caso contrario se convertirían en uno más y las condiciones de dicho mercado, en este caso el financiero, no cambiarían.

En el caso del Biess, este cuenta con un fondeo de largo plazo, es decir con reservas técnicas que debe devolver en un plazo extendido lo que le genera condiciones ideales para el otorgamiento de un crédito de largo plazo como el de la vivienda.

Los principios en los que debe basarse la inversión de las reservas de la protección social son seguridad, rendimiento y utilidad económica y social (Cichon et al., 2006). La seguridad es indispensable en el manejo de recursos de terceros, evitando elementos especulativos (2006, pp. 460-461). En términos del rendimiento, "el beneficio ofrecido debería regir la elección de activos comparables en los que invertir las reservas de la seguridad social" sobre todo para las reservas técnicas (2006, p. 461).

Respecto del tercer principio, se recuerda que:

Los fondos de pensiones (...) pueden contribuir a fomentar la producción nacional, puesto que, a diferencia de otros inversores, tienen a su disposición, no sólo un gran volumen, sino también un horizonte de largo plazo para su inversión. Si estos medios se emplean estratégicamente, pueden ayudar a garantizar una base de altos ingresos de la economía. (2006, p. 461)

El objetivo en el uso de las reservas es buscar un justo equilibrio entre la maximización del rendimiento y la minimización del riesgo, optimizando el horizonte temporal entre los dos (2006, p. 462).

La metodología de este artículo es explicativa e inferencial, la cual es válida para un tema poco estudiado (Hernández Sampieri, Fernández Collado, \& Baptista Lucio, 2010, p. 97). Los instrumentos de investigación uti- 
lizados serán las encuestas de empleo del Instituto Nacional de Estadística y Censos (INEC), las de oferta y demanda de crédito del Banco Central del Ecuador (BCE), así como los registros de la cartera y las tasas de interés de la misma institución. La limitación está en algunos vacíos en las series de información del Biess.

Es preciso aclarar que el artículo realizará comparaciones con la banca privada pero siempre teniendo en cuenta que los objetivos que persigue cada una son distintos, la privada busca rentabilidad financiera y la pública o de desarrollo, no la omite pero prioriza la rentabilidad social, por ejemplo con el acceso a vivienda propia.

\section{Resultados}

En el 2009 la economía ecuatoriana se vio afectada por la crisis económica mundial y, por tanto, la banca privada restringió su oferta de crédito manteniendo comportamientos procíclicos en la fase baja del ciclo económico (Allen \& Saunders, 2004), mientras que la banca pública aumentó las opciones de crédito. El Biess empezó a funcionar en octubre 2010 pero el IESS ya había iniciado su actividad crediticia unos meses antes. Estos dos hechos incrementaron la competencia en el sector bancario y generaron inquietud en las IFI's privadas (King \& Jácome, 2013, pp. 95-96). Las preocupaciones provenían del fondeo de largo plazo del Biess, así como del hecho de que iba a dejar de comprar papeles financieros.

La ventaja fundamental de irrumpir en colocaciones directas y no ser sólo una banca de segundo piso es que el Biess podía obtener mayor rentabilidad que la que tenía con la compra de papeles privados y públicos en el mercado de valores y, además, puede cobrar las cuotas más fácilmente en descuentos en roles para el repago de dichas colocaciones.

Para situarnos adecuadamente en el contexto de la época, otro hecho importante se dio en agosto del año 2007, cuando se aprobó la "Ley de Justicia Financiera" para fijar techos a las tasas de interés activas. Si bien la Ley fue declarada inconstitucional en esta parte, el directorio del Banco Central definió techos máximos referenciales por segmentos (Astorga \& Morillo, 2010, p. 6).

En el gráfico 1 se presenta la evolución de las tasas efectivas activas máximas producto de la regulación. Estas se definen según el BCE (2010a) 
como aquellas que el Directorio del Banco Central permite en función de un factor que multiplica la tasa de las últimas cuatro semanas a la fecha de la regulación.

Mientras que la tasa referencial, se define como el promedio ponderado por monto de las operaciones realizadas por el sistema financiero privado en las últimas cuatro semanas (BCE, 2010a).

\section{Gráfico 1}

\section{Tasa activa máxima y tasa referencial para el segmento de vivienda entre agosto 2007 a abril 2009 (en porcentaje)}

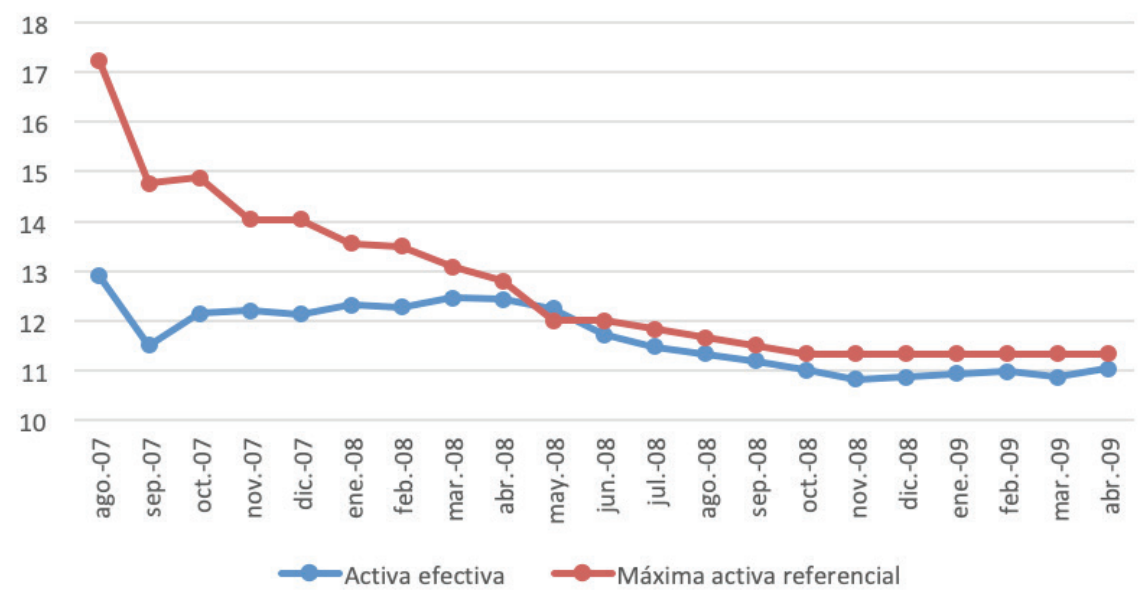

Fuente: BCE (2010a).

Elaboración: Propia.

Entonces, antes de la aprobación oficial de la Ley de creación del Biess, se produjo una reducción en la tasa máxima activa permitida para el segmento de vivienda. En agosto 2007 esta era de 17,23\%, al mes siguiente pasó a $14,77 \%$ y se fue reduciendo paulatinamente hasta que, a partir de octubre 2008, la tasa máxima referencial para crédito hipotecario se mantuvo en $11,33 \%$. En este mismo período, agosto 2007-octubre 2008 la tasa activa efectiva pasó de $12,9 \%$ a $11 \%$, es decir se produjo una reducción de casi dos puntos en la tasa cobrada. 
La política de fijación de techos en las tasas de interés por segmento de crédito generó fuerte crítica por parte de los bancos privados, argumentando incluso que ocurriría represión financiera (Astorga \& Morillo, 2010), es decir que se reduciría el crédito en la economía, cosa que no ocurrió como se verá más adelante.

En el primer trimestre del 2010, una tercera parte de las IFI's tenía condiciones menos restrictivas en los créditos de vivienda (BCE, 2010b). Entre estos, se encontraba el Banco del Pacífico que opera como banco privado pero que en ese momento era de propiedad del Banco Central y que tenía programas específicos de créditos de vivienda como el Hipoteca Pacífico, que aumentó las opciones de financiamiento de vivienda

Todo esto fue poco a poco dinamizando el crédito hipotecario en operadores privados y públicos y, hacía que a fines del 2011, el Biess registre una participación del $62 \%$ en la generación de la cartera hipotecaria total (BCE, 2012, p. 46), situación que iba de la mano de crecimientos económicos en esos años.

Además de la colocación directa por parte del Biess, se realizaron compras de cartera hipotecaria de vivienda a la banca privada, que le otorgaban mayor liquidez a esta para colocar este u otro tipo de créditos. Del otro lado, esto era conveniente para los afiliados porque recibían mejores condiciones que las ya obtenidas en la banca privada, con una tasa efectiva anual entre 8,19 y 9,04 (Biess, 2018) y para el Biess significaba mejorar los rendimientos obtenidos en papeles públicos o en el mercado de valores.

En el año 2008, el IESS compró un 44\% de obligaciones financieras como certificados de depósito, certificados de inversión y titularizaciones crediticias y destinó el $42 \%$ a papeles públicos (Superintendencia de Compañías, 2008, sec. 37). Un certificado de depósito, principal título de renta fija que compraba el IESS tiene un rendimiento actual en torno al $6 \%$ en el plazo más largo, es decir solo en esos papeles se podía obtener un aumento en rentabilidad bruta de 3 puntos. Mientras que los bonos del Estado que fueron emitidos en diciembre 2008 y comprados por el IESS, tuvieron un rendimiento del 6,63\% (Ministerio de Finanzas, 2008, p. 5).

Esta política impulsó el mercado inmobiliario en construcción de viviendas, entre el 2008 y el 2012, hubo un crecimiento del 134\% en el número de viviendas por construirse (Ordóñez, 2014, p. 17).

Estos cambios en el mercado hipotecario despertaron otra alerta por parte de un miembro de la Asociación de Bancos, cuando en marzo 2011 declaró el peligro de una posible burbuja inmobiliaria en el país (EFE, 2011). 
Los argumentos esgrimidos daban cuenta de la preocupación que generaba el nuevo actor y que podría inundar de crédito a la economía, así como el hecho de que se otorgaba el 100\% del valor de la vivienda como crédito. En este caso, la persona del gremio bancario daba cuenta de la burbuja por el aumento de los precios de la vivienda más no por problemas en la concesión del crédito.

Ecuador, tenía presente la experiencia hipotecaria que provocó la crisis económica mundial y no se relajaron las condiciones en el otorgamiento del crédito. Lo que era cierto es que el Biess dejaba de utilizar recursos de las reservas para la compra de instrumentos financieros y eso generaba malestar en los bancos.

También cabe indicar que las condiciones económicas fueron cambiando tanto en la oferta como en la demanda. En el año 2015, existió una importante reducción del precio del petróleo que afectó la liquidez de la economía así como las cuentas fiscales, un año después, en el 2016, se produjo un terremoto en Manabí que repercutió de forma negativa en la actividad económica del país. En ese año también se produjo el anuncio de dos leyes, la de plusvalía, aprobada en diciembre 2016 y la de herencias, para la cual sólo se aprobó una norma anti-elusiva en junio 2016. Estos anuncios influyeron en la actividad en el sector de la construcción. La Ley de Plusvalía se derogó en el 2018.

Es necesario indicar que el Biess otorga financiamiento no sólo para primera vivienda, sino que lo hace para: vivienda terminada, construcción de vivienda, remodelación y ampliación, sustitución de hipoteca, terrenos y construcción, otros bienes inmuebles y vivienda hipotecada.

A continuación, se presentan algunos de los resultados a raíz de la aparición del Biess:

\section{Aumento de la cartera hipotecaria privada}

El primer efecto, contrario a lo que alertaban actores financieros privados tiene que ver con el hecho de que la cartera hipotecaria privada se mantuvo e incluso creció a partir del 2017. En el gráfico 2 se observa el monto total de las operaciones activas de las IFI's privadas y la respectiva tasa promedio efectiva. 


\section{Gráfico 2}

Monto anual de operaciones activas hipotecarias privadas y tasa promedio anual activa entre 2009 y 2018 (en millones de dólares y porcentaje)

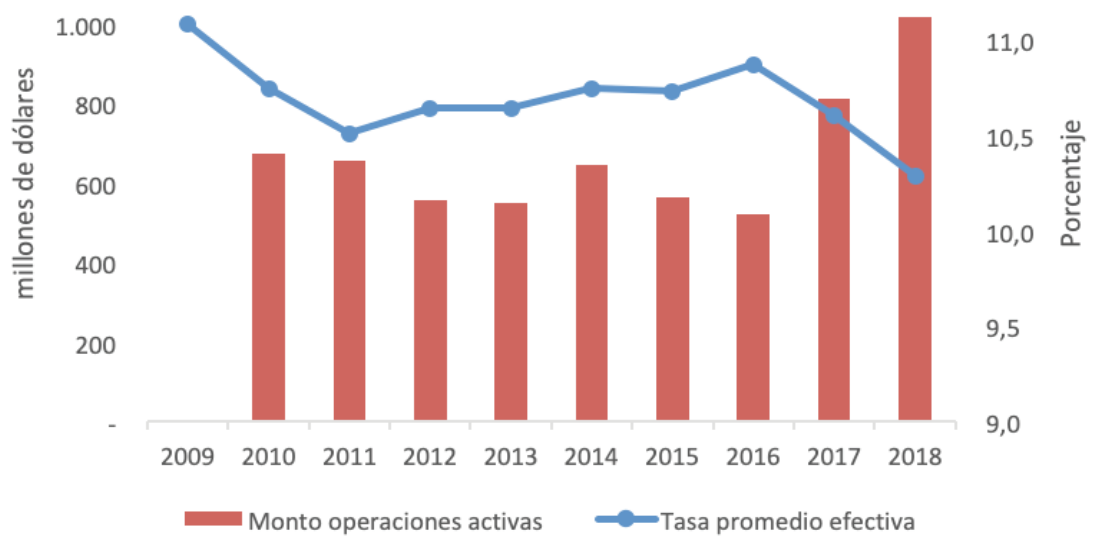

Fuente: BCE (2019).

Elaboración: Propia.

La banca privada no dejó de mantener cartera hipotecaria, parte de esta cartera fue comprada por el Biess y conservó montos de colocación anuales superiores a los 650 millones de dólares anuales entre 2010 y 2011. Entre el 2012 y 2015 la cartera originada por IFI's privadas se redujo y tuvo un comportamiento oscilante hasta el 2016 con el nivel más bajo por los problemas económicos expuestos anteriormente.

En el gráfico anterior, es notable como el momento en que las IFI's privadas aumentan el monto de operaciones activas a partir del 2017, la tasa promedio efectiva se reduce. Por tanto, además de la reducción de la tasa por efecto de los techos hasta el 2008, se encuentran tres períodos en la evolución de la tasa efectiva: primero de reducción hasta el 2011, segundo de crecimiento entre 2012 y 2016 y tercero de reducción a partir del 2017. En el primer y tercer períodos se observa que a mayores montos de operaciones activas de parte de los privados, menor es la tasa. Esto resulta más evi- 
dente con el aumento de la cartera en los años 2017 y 2018 que podría ser explicado por razones políticas en un período en el que las IFIS mantenían una mejor relación con el gobierno de turno y recibieron algunas prebendas (King \& Samaniego, 2019). En estos años el Biess reducía su participación del 66\% al $43 \%$ entre el 2016 y 2018 y la sustitución de hipoteca se encontraba en niveles bajos, lo cual es lógico porque la tasa del segmento hipotecario era menor y había menos interés en sustituir hipoteca que cuando recién entró a operar el Biess.

\section{Reducción de la tasa en el segmento hipotecario}

En base a lo anterior se resume la evolución de la tasa en este segmento en dos momentos, una caída de 1,87 puntos porcentuales entre agosto 2007 y abril 2009 por la política de techos a las tasas de interés. El otro momento ocurrió con la presencia del Biess, en el que la tasa entre mayo 2009 y diciembre 2018 tuvo una disminución de 2,22 puntos porcentuales con comportamientos erráticos en el intermedio, tal como se explicó antes.

En el segundo momento la reducción fue mayor pese a que el sector inmobiliario pasó por un mal momento en los años 2015 y 2016, producto de la recesión económica, un terremoto y el anuncio de medidas con incidencia en el sector. Esto ocurrió sin represión financiera en la cartera total y menos en el segmento de ingresos más bajos que no es de interés de la banca privada para que el que se planteó otra medida que será mencionada en el siguiente acápite.

\section{Efectos en la economía real de las reservas por parte del Biess}

Esto atiende al principio de la suficiencia de la seguridad social, ya que representa una fuente notable de ahorro en las economías y que, por tanto, son recursos apetecidos por su magnitud y por la característica de la temporalidad en fondeo.

Los usos alternativos de las reservas técnicas de la seguridad social son de especial importancia por sus efectos en la economía real y definirán la prioridad política en la asignación de estos. El ahorro es también una variable necesaria a nivel macroeconómico para impulsar la inversión.

La seguridad social recauda cotizaciones y tiene obligaciones que son menores. Esta es la fase de acumulación del sistema, antes de que este madure 
cuando la población envejece y aumenten las obligaciones con los jubilados o el número de afiliados activos se reduzca (Cichon et al., 2006, p. 523).

\section{Acceso a la vivienda propia}

El acceso a la vivienda adecuada es considerado un derecho humano fundamental a partir de 1948 (Habitat, 2010). En términos del acceso a vivienda propia, a diciembre del 2007 un 65,9\% de las viviendas se encontraba en un régimen de tenencia propia y un 18,9\% en arriendo en la que casi no había diferencias entre viviendas con alguna persona afiliada y sin ella. Mientras que entre los hogares con vivienda propia, el porcentaje era de $68,2 \%$ para los que tenían algún afiliado y de 64,5\% para los que no. Los hogares con vivienda cedida eran menores entre aquellos con alguna persona afiliada, $10,5 \%$ respecto a $14,2 \%$ que no. Para este mismo año, el $75 \%$ de los hogares del primer decil por ingresos contaba con vivienda propia y el $73 \%$ para el segundo decil, siendo los deciles con porcentajes más altos y estos porcentajes son mayores entre los hogares no afiliados. Asimismo, el porcentaje de vivienda arrendada era de $8 \%$ en el primer decil y $9 \%$ en el segundo (cálculos propios en base a INEC, 2007).

Es decir, si bien el Biess podía otorgar créditos solamente a sus afiliados, la diferencia de hogares con vivienda propia era 3,7 puntos y los dos primeros deciles contaban con menores porcentajes de viviendas en arriendo. Al 2018, en cambio se encuentra que a nivel nacional el porcentaje de hogares con vivienda propia subió a 69,5\% y el de arriendo bajó a 17,5\%. En estos años, existieron además programas específicos que proporcionaron vivienda propia a los estratos más bajos por lo cual es importante separar los resultados entre hogares con alguna persona afiliada y los que no (cálculos propios en base a INEC, 2018).

Al desagregar la información de tenencia de vivienda entre afiliados y no afiliados para el 2018 se encuentra que los hogares con alguna persona afiliada aumentaron más la tenencia de vivienda propia, con $71,9 \%$ respecto al $66,6 \%$ que no tenían.

Los cambios entre el 2007 y el 2018 se presentan en la tabla 1, en la cual se observa que los hogares con alguna persona afiliada reducen el porcentaje de viviendas arrendadas en 1,7 puntos, en las cedidas en 0,9 y aumentan en 3,7 la tenencia de vivienda propia. 
Tabla 1

Cambio en la tenencia de vivienda de los hogares por afiliación a la seguridad social entre 2007 y 2018 (en puntos porcentuales)

\begin{tabular}{|l|c|c|}
\hline & No afiliados & Afiliados \\
\hline Arriendo & $-1,0 \%$ & $-1,7 \%$ \\
\hline Propia & $2,1 \%$ & $3,7 \%$ \\
\hline Cedida & $0,3 \%$ & $-0,9 \%$ \\
\hline
\end{tabular}

Fuente: INEC (2007), INEC (2018).

Elaboración: Propia.

Los quintiles que más aumentaron su participación entre los hogares con vivienda propia y alguna persona afiliada fueron los 2 y 3 , lo que también se explica por el aumento de personas con afiliación en estos estratos de ingresos.

\section{Resultados negativos, falta de gestión de cobranza y situación económica adversa}

Finalmente, uno de los problemas con los que se ha enfrentado el Biess ha sido la morosidad en los últimos años en que la actividad económica se ha visto afectada, principalmente a partir del año 2016.

\section{Tabla 2}

Tasa de morosidad en los créditos hipotecarios del BIESS entre 2012 y 2018 (en porcentaje)

\begin{tabular}{|c|c|c|c|c|c|c|}
\hline $\mathbf{2 0 1 2}$ & $\mathbf{2 0 1 3}$ & $\mathbf{2 0 1 4}$ & $\mathbf{2 0 1 5}$ & $\mathbf{2 0 1 6}$ & $\mathbf{2 0 1 7}$ & $\mathbf{2 0 1 8}$ \\
\hline 1,0 & 1,7 & 2,0 & 2,9 & 5,3 & 6,1 & 6,5 \\
\hline
\end{tabular}

Fuente: González, J. (2019).

Elaboración: Propia.

La información disponible es menos amplia en este ámbito por lo que se desconoce si esta morosidad es un fenómeno puntual en un área geográfica o es más amplio. Está claro que frente a situaciones económicas adversas, 
las personas pierden sus trabajos y dejan de pagar los préstamos. Al parecer, también han existido problemas en la concesión del cupo de crédito (González, P., 2019b) que se debió a la injerencia de algunos constructores para facilitar la compra de sus inmuebles.

El índice de morosidad (Tabla 2) se encuentra por encima del 6\% en el 2018. Este indicador no puede ser comparado con el de la banca privada porque se conoce que el BIESS no ha castigado su cartera y por tanto, el índice de morosidad del Biess incluye cartera que debería estar castigada (Biess, 2019). En el caso de la banca privada, los índices de morosidad excluyen la cartera castigada. Se sabe que no se han efectuado gestiones de castigo de cartera "por limitaciones operativas y contables" Biess (2019) a las que pueden añadirse razones políticas de realizar gestiones de cobranza y administración de cartera en riesgo, así como la injerencia antes mencionada en la determinación del cupo.

\section{Posible burbuja inmobiliaria}

Macías Rendón, Guzmán Sigüencia, y Ramírez Delgado (2015) encuentran que entre enero de 2008 y octubre 2014 existió un incremento en el precio de la vivienda de $1,68 \%$ promedio anual $(2015$, p. 60$)$ y se identifica que el precio de la vivienda creció más que el precio del alquiler. Sin embargo, los autores encuentran que las variaciones en el precio de la vivienda se explican en un $93 \%$ por variables fundamentales de oferta (oferta de salarios y costos de la construcción) y demanda (por la actividad económica), por tanto descartan que haya existido una burbuja inmobiliaria en Ecuador (2015, p. 62).

\section{Discusión y avances}

La presencia del Biess y la compra inicial de cartera modificó el mercado financiero e inmobiliario en el Ecuador. Se aplicaron distintas medidas de regulación financiera en el segmento de vivienda. La primera de techos máximos tuvo un efecto inicial de reducción en la tasa promedio del hipotecario que bajó de $12,9 \%$ a $11,03 \%$ entre agosto 2007 y abril 2009, es decir 1,87 puntos.

Una vez que el Biess entra a operar, la tasa continúa reduciéndose hasta ubicarse en 9,55\% en enero 2019, rompiendo por primera vez el piso del 10 
por ciento. Así entre mayo 2009 y diciembre 2018 la tasa en este segmento tuvo una disminución de 2,22 puntos porcentuales con comportamientos erráticos en el intermedio.

Cabe anotar que este artículo no considera a la provisión directa como un fin en sí mismo, ya que el Biess como plantea la Ley de su creación buscaba generar empleo y valor agregado, pero además generó las condiciones para que la tasa se reduzca en el segmento hipotecario.

La utilización de las reservas técnicas de la seguridad social en el mercado hipotecario local tiene algunas ventajas para la misma sostenibilidad de la seguridad social al obtener mejores retornos (en torno a tres puntos) y en el calce de plazos mediante préstamos de largo plazo para vivienda con un fondeo en el mismo plazo.

La provisión directa dinamizó la economía real a través del impulso al mercado inmobiliario, a diferencia de lo que sucedía en el pasado cuando los recursos de las reservas se usaban en la compra de papeles financieros de renta fija con plazos menores que no se traducían en mejores condiciones de financiamiento.

Es importante resaltar que un operador público puede ser promisorio si este operador se convierte en un competidor de la banca privada ofreciendo mejores condiciones financieras, como fue el caso del Biess.

Sin embargo, es preciso mencionar algunos problemas de la política ya que no todo el crédito fue para la primera vivienda y el primer quintil no fue uno de los mayores beneficiarios por la misma estructura de la seguridad social y su relación con el mercado de trabajo formal. Es por lo que, en agosto 2015, se crea el segmento de vivienda de interés público que es el otorgado a créditos "con garantía hipotecaria a personas naturales para la adquisición o construcción de vivienda única y de primer uso, concedido con la finalidad de transferir la cartera generada a un fideicomiso de titularización" (BCE, 2019).

La burbuja inmobiliaria alertada por los bancos no existió porque la oferta aumentó de forma importante aunque en algunos lugares el valor del $\mathrm{m}^{2}$ sí aumentó y no se debe dejar de tener en cuenta experiencias de burbujas especulativas en el mercado inmobiliario (Harvey, 2014, p. 92).

Existe un balance positivo, no perfecto, en la política de provisión directa con el uso de las reservas técnicas de la seguridad social. Esto generó cambios en el mercado hipotecario, política que se mantiene hasta la fecha con ciertas modificaciones en el cálculo del cupo del crédito de acuerdo a menores porcentajes del ingreso y el avalúo (P. González, 2019b). Estas re- 
formas podrían estar justificadas en el marco de la capacidad razonable de pago del ingreso (Hudson, 2018, p. 383) sin embargo también deben ser leídas como una forma de liberar recursos para financiar el mercado de valores (J. González, 2019) y préstamos quirografarios (Lizarzaburu, 2019). Estos últimos no tienen una incidencia directa en la generación productiva y podrían terminar en manos de la banca privada para pagar obligaciones de consumo contratadas previamente.

La obtención de recursos en el largo plazo es crucial para la economía real, sobre todo para una estrategia de cambios estructurales que necesitan tiempos largos para que ocurran (Mazzucato, 2013, p. 51). La vivienda ha sido analizada en este artículo, pero puede haber otros sectores con mayores efectos multiplicadores en el largo plazo que podrían ser analizados.

\section{Conclusiones}

La provisión directa ha sido en Ecuador una de regulación alternativa con el fin de mejorar las condiciones de financiamiento en el segmento hipotecario. Esto implicó, pasar de una situación ineficiente en la cual los recursos de las reservas se invertían en productos financieros que no mejoraban las condiciones financieras crediticias.

Antes de la irrupción del Biess, en el Ecuador se definieron precios máximos por segmentos de crédito que redujeron todas las tasas activas. La puesta en marcha de un banco público que opere con recursos de la seguridad social partió de un cambio constitucional y legal con resistencia de parte de operadores privados. Es preciso mencionar que, ante cambios en el mercado financiero, los bancos privados hacen presión y exageran sobre los posibles efectos negativos como se ha ejemplificado en este artículo.

En todo el segmento hipotecario, se produjo una notable reducción de la tasa por efecto del control a las tasas y de la aparición del Biess en el mercado. Asimismo, los afiliados mejoraron las condiciones de sus créditos. Esto tuvo un efecto social en el acceso a la vivienda propia, más pronunciada para los quintiles 2 y 3 . En términos económicos esto representó un aumento de la liquidez que se canaliza hacia la economía real con fondos de largo plazo. Como desventaja se encuentra la injerencia de los promotores inmobiliarios y los índices de morosidad que pese a no ser comparables con los de la banca privada deben ser atendidos. 
Si bien no se cuenta con la información detallada de rentabilidades promedio por producto del Biess, en los papeles en los que invertía el Biess sí se encuentra un mejor rendimiento bruto con mejores garantías y calce de plazos.

Se cumple la hipótesis respecto a la reducción de la tasa en el segmento hipotecario, sin embargo, los efectos de la regulación en los precios máximos y del nuevo operador público se retroalimentan uno al otro. Respecto de la vivienda propia, esta aumentó en 3,6 puntos a nivel nacional y en 3,7 puntos porcentuales para los hogares con personas afiliadas, por tanto sí se cumple la hipótesis.

Las políticas promercado invisibilizan experiencias alternativas como esta al poner énfasis sólo en las dificultades y este artículo aporta a la discusión práctica del legado de las políticas alternativas de regulación con operadores públicos en base a la información disponible.

\section{Agradecimientos}

Una versión preliminar de este artículo se presentó en el XXXVII Congreso Internacional de la Asociación de Estudios Latinoamericanos (LASA) en Boston, Estados Unidos, congreso para el cual la autora recibió una beca de viaje para su participación. Se agradecen los comentarios y observaciones de Pedro Montes, Pablo Samaniego y dos lectores ciegos.

\section{Bibliografía}

Allen, L., \& Saunders, A. (2004). Incorporating Systemic Influences Into Risk Measurements: A Survey of the Literature. Journal of Financial Services Research, 26(2), 161-191. https://doi.org/10.1023/ B:FINA.0000037545.38154.8a

Asamblea, C. (2008). Constitución de la República del Ecuador. Montecristi: Registro Oficial.

Astorga, A., \& Morillo, J. (2010, mayo). Acceso al crédito en el Ecuador; ¿retorno a la represión financiera? Centro de Investigaciones Económicas. Universidad de las Américas, 2. Recuperado de: https://bit.ly/2Qq7Se3 (2019-04-06).

Banco Central del Ecuador-BCE (2010a). Información Estadística Mensual (Núm. 1906). Recuperado de: https://bit.ly/2dROgA0 (2019-03-20). 
(2012). Evolución del crédito y tasas de interés efectivas referenciales [Boletín Mensual]. Recuperado de: https://bit.ly/2d5wnJk (2019-04-11).

(2019). Evolución del Monto de las operaciones activas y pasivas del Sistema Financiero Nacional Boletín Mensual (Boletín Mensual Núm. 89). Recuperado de: https://bit.ly/2d5wnJk (2019-04-11).

Biess (2011). Plan estratégico del Banco del Instituto ecuatoriano de la Seguridad Social. Resolución BIESS-016-2011.

(2017, diciembre). Plan estratégico institucional 2016-2020. Resolución 024-2017.

(2018). Tasas de interés Tarifario. Recuperado de: https://bit.ly/2Hx32Y6 (2019-02-19).

(2019). Consultas con funcionario del Biess.

Carrillo, D. (2013). Análisis de la Influencia de los préstamos hipotecarios otorgados por el Instituto Ecuatoriano de Seguridad Social (IESS).Banco del Instituto Ecuatoriano de Seguridad Social (BIESS), sobre el crecimiento del sector de la construcción, durante el período 2007-2011. (Tesis Pregrado) Pontificia Universidad Católica del Ecuador, Facultad de Economía. Recuperado de: https://bit.ly/32hkzNW

Cichon, M., Scholz, W., Meerendonk, A. van de, Hagemejer, K., Bertranou, F., Plamondon, P., \& Spanien (Eds.) (2006). Financiación de la protección social. Madrid: Ministerio de Trabajo y Asuntos Sociales.

Deere, C. D., \& Catanzarite, Z. B. (2017). ¿Quién obtiene préstamos para acumular activos? Clase, género y endeudamiento en el mercado crediticio del Ecuador. Revista CEPAL, 122, 115-137.

DNTI (2009, mayo 11). Registro Oficial No. 587. Registro Oficial del Ecuador. Recuperado de: https://bit.ly/2HB6S3Y

EFE, A. (2011, marzo 24). Banca privada alerta de inicio de una posible burbuja inmobiliaria en Ecuador. El Universo. Recuperado de: https://bit. ly/2EJzd6v (2019-05-15).

González, J. (2019, enero 25). Más de \$4 mil millones invertirá el BIESS en 2019. El Universo. Recuperado de: https://bit.ly/2RhTJOI (2019-05-15).

González, P. (2019a, febrero 23). La morosidad de los hipotecarios del BIESS alcanzó 6,49\%; la de la banca privada es de 2,78\%. El Comercio. Recuperado de: https://bit.ly/2GZaXik (2019-05-15).

(2019b, mayo 11). Cambios en hipotecarios aplican en seis meses. El Comercio. Recuperado de: https://bit.ly/2HdRNoQ 
Habitat, O. (2010). El derecho a una vivienda adecuada. Folleto informativo, 21. Recuperado de: https://bit.ly/2O9AZn9

Harvey, D. (2014). 17 contradicciones y el fin del capitalismo. Quito: IAEN.

Hernández Sampieri, R., Fernández Collado, C., \& Baptista Lucio, P. (2010). Metodología de la investigación (Sexta). México: McGraw-Hill.

Hudson, M. (2018). Matar al huésped: Cómo la deuda y los parásitos financieros destruyen la economía global. Madrid: Capitan Swing.

Instituto Nacional de Estadísticas y Censos, INEC (2007, diciembre). Banco de Información estadística. Recuperado de: https://bit.ly/1YRnioF (2019-04-06).

(2008, diciembre). Banco de Información estadística. Recuperado de: https:// bit.ly/1YRnioF (2019-04-06).

(2009, diciembre). Banco de Información estadística. Recuperado de: https:// bit.ly/1 YRnioF (2019-04-06).

(2018, diciembre). Banco de Información estadística. Recuperado de: https:// bit.ly/1 YRnioF (2019-04-06).

King, K., \& Jácome, H. (2013). El sector financiero: solidez gracias a la liquidez. En Cuadernos de trabajo. Situación económica y ambiental del Ecuador en un entorno de crisis internacional. Recuperado de (https://bit. ly/2M9yEss).

King, K., \& Samaniego, P. (2019). A río revuelto, ganancias de varios pescadores. Ecuador Debate, (106).

Lizarzaburu, G. (2019, mayo 10). El BIESS aumenta el monto de los préstamos quirografarios. Expreso. Recuperado de: https://bit.ly/2VPBJNS

Macías Rendón, W., Guzmán Sigüencia, L., \& Ramírez Delgado, M. (2015). ¿Existe evidencia de burbuja inmobiliaria en el Ecuador? PODIUM, (28), 5568. https://doi.org/10.31095/podium.v0i28.33

Mazzucato, M. (2013). The entrepreneurial state: debunking public vs. private sector myths. London: Anthem Press.

Micco, A., Panizza, U., \& Levy, Y. (2007). A Reappraisal of State-Owned Banks. Economía, 7(2). https://doi.org/10.1353/eco.2007.0015

Ministerio de Finanzas (2008). Boletines de Deuda Interna. Boletín estadístico 48. Recuperado de: https://bit.ly/2Xz260p (2019-05-04).

Ordóñez, M. (2014, octubre 15). El Biess marcó la pauta inmobiliaria. Revista Gestión, (244).

(2015). Impacto de la creación del Banco del Instituto Ecuatoriano de Seguridad Social (BIESS) sobre la oferta de crédito de vivienda de bancos privados y mutualistas y sobre el sector inmobiliario del Ecuador, perío- 
do 2008-2012 (Pregrado). Pontificia Universidad Católica del Ecuador, Facultad de Economía. Recuperado de: https://bit.ly/2LGyXZY

Sanguinetti, P., Arreaza, A., Rodríguez, P., Álvarez, F., Ortega, D., \& Penfold, M. (2011). Servicios financieros para el desarrollo. Promoviendo el acceso en América Latina. Recuperado de: https://bit.ly/2S9h8Em

Secretaría Nacional de Planificación y Desarrollo, SENPLADES (2007). Plan Nacional de Desarrollo 2007-2010. Planificación para la revolución ciudadana. Quito: Secretaría Nacional de Planificación y Desarrollo, SENPLADES.

(2009). Plan Nacional para el Buen Vivir, 2009-2013: construyendo un estado plurinacional e intercultural. Quito: Secretaría Nacional de Planificación y Desarrollo, SENPLADES.

Serrano Cueva, O., \& Naranjo Peñaherrera, M. P. (2014). El banco del IESS: crónica de su creación. Quito: Abya-Yala.

Stiglitz, J. (2000). La economía del sector público (3ra ed.). Barcelona: Antoni Bosch.

Subirats, J., Knoepfel, P., Larrue, C., \& Varonne, F. (2008). Análisis y gestión de políticas públicas. Barcelona: Ariel.

Superintendencia de Compañías (2008). Anuario Estadístico. Mercado de Valores [Consulta]. Recuperado de: https://bit.ly/2S5fq6Q (2019-05-03).

Fecha de envío: 2019/05/25; Fecha de aceptación:2019/07/09;

Fecha de publicación: 2019/09/01 